\title{
Towards clinical application of 7T TOF angiography
}

\author{
Sebastian Schmitter ${ }^{*}$,Edward J Auerbach, Gregor Adriany, Kamil Ugurbil, Pierre-Francois Van de Moortele \\ From 15th Annual SCMR Scientific Sessions \\ Orlando, FL, USA. 2-5 February 2012
}

\section{Background}

As shown at 7T Time-of-Flight (TOF) angiography significantly benefits from ultra high field (UHF) [1,2], because of shorter $\mathrm{T} 1$ relaxation constant and increased SNR. However, significant challenges have to be overcome before using 7T TOF in clinical applications: I) RF induced specific absorption rate (SAR) substantially increases at UHF, preventing the use of standard RF pulses (venous Saturation (SAT), Magnetization Transfer $(\mathrm{MT}))$ to improve TOF contrast. II) Severe transmit $\mathrm{B} 1(\mathrm{~B} 1+)$ heterogeneity at $7 \mathrm{~T}$ impairs contrast homogeneity.

\section{Methods}

In this work we approached both challenges with complementary strategies:

1) SAR reduction with VERSE [3,4] for excitation and travelling venous SAT pulses. The max amplitude of VERSE RF pulses was set to a fraction $\kappa$ EXC (or $\kappa S A T$ ) of the max amplitude of initial excitation (or SAT) pulse. 2) Sparse application of MT [5] (10\% of the centered k-space lines in phase and slab direction [6]).

3) Improved homogeneity of RF excitation profile with B1+ shimming (16 transmit channels), using fast multi channel B1+ calibration [7]. The standard deviation divided by the mean (std/mean) of $\mathrm{B} 1+$ was minimized over the center slices of 3 TOF slabs using nonlinear optimization algorithms.

\section{Results}

1+2) Fig.1 shows maximum intensity projections (MIPs) for a) the original sequence with $\kappa \mathrm{EXC}=100 \%$ (no VERSE), without SAT and without MT, and b) our modified sequence with $\kappa \mathrm{EXC}=50 \%$, with venous $\mathrm{SAT}$ $(\kappa \mathrm{SAT}=25 \%)$ and sparse MT. Quantitative results: vessel

\footnotetext{
University of Minnesota, Center for Magnetic Resonance Research,
} Minneapolis, MN, USA to tissue contrast improved by $>50 \%$, venous signal suppressed by $\sim 90 \%$. Strong fat signal suppression observed within the excitation slab results from the large spatial chemical shift of VERSE-SAT pulse. SAR values were, for a) and b), $17 \%$ and $69 \%$ of maximum SAR limit, respectively. By comparison, using standard excitation, SAT and MT RF pulses (no VERSE/no sparse RF) would amount $319 \%$ of SAR limit.

3) Homogeneity strongly improved with B1+ shimming (Fig.2), with std/mean reduced by a factor 2.2 . Smaller vessels in the brain periphery show higher contrast or become visible (see arrows). In general, however, spatial homogeneity optimization reduces B1+ efficiency, thus increases SAR. For example, for the excitation pulse SAR with B1 shimming was 4.7 times the value without B1 shimming, however this pulse represents only a fraction of SAR.

\section{Conclusions}

We demonstrate, with appropriate RF pulse design, substantial gains in contrast and B1+ homogeneity for TOF angiography at $7 \mathrm{~T}$. These results strongly support the potential of expanding non-contrast enhanced angiography at $7 \mathrm{~T}$ towards clinical investigations.

\section{Funding}

ACKNOWLEDGEMENT: DFG: SCHM 2677/1, NIH P41 RR008079, P30 NS057091, R01 EB000331, NIH R21 EB009133; Keck Foundation.

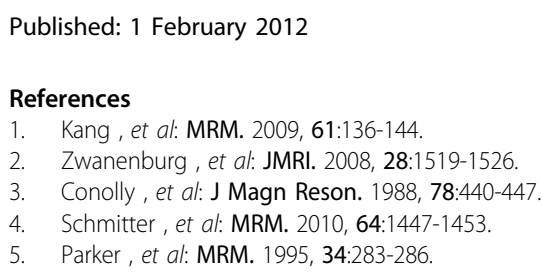




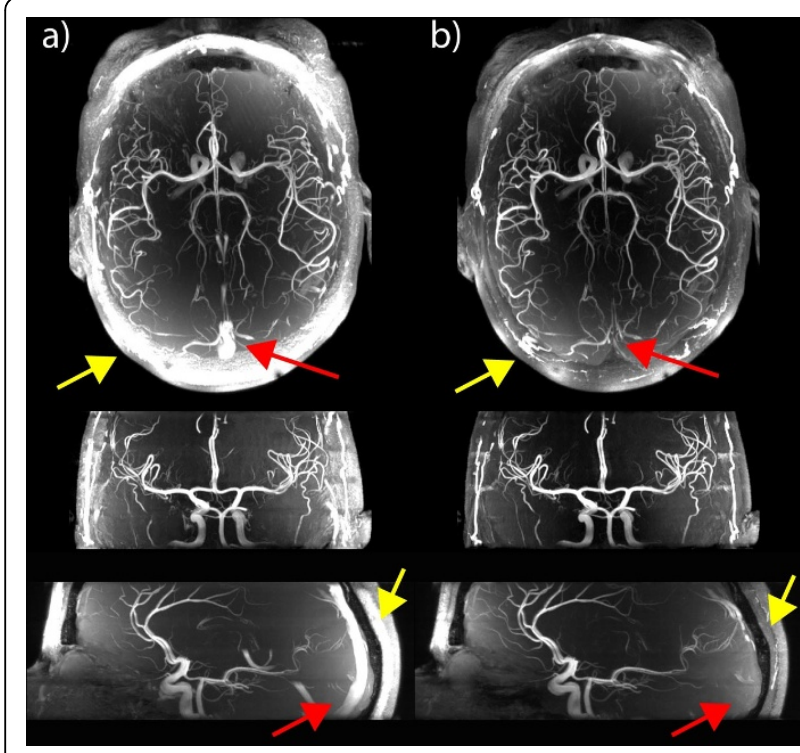

Figure 1 MIP images of the original TOF (a) without VERSE, venous SAT or MT and the modified TOF sequence (b) with venous SAT and with MT acquired at 7 Tesla. Successful suppression of venous signal (red arrows), significant fat signal supression (yellow arrows) as well as improved overall background suppression is visible in b). Images were not corrected for receive profile for better visualization of the individual effects.

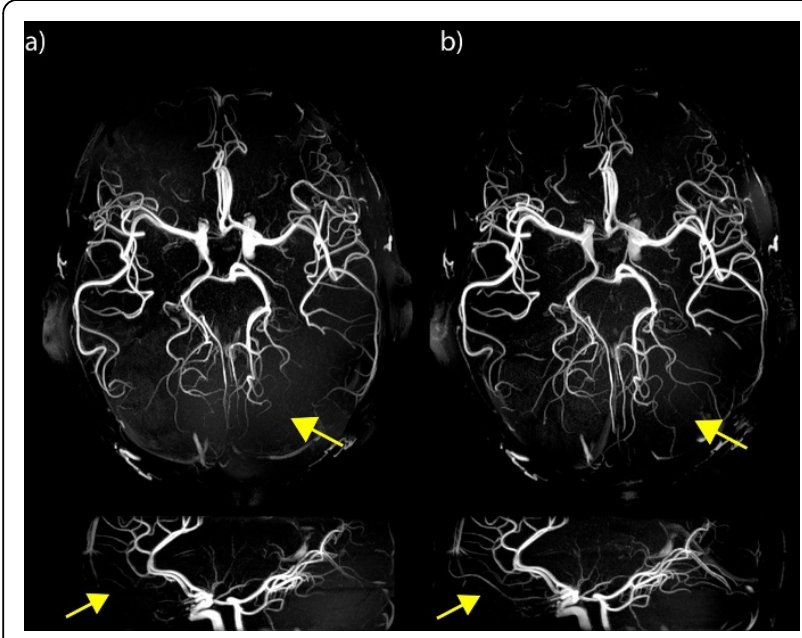

Figure 27 Tesla TOF datasets with a) circular polarized RF mode used for excitation and venous saturation, b) CP mode used for saturation and B1 phase shimming for optimized homogeneity used for excitation pulses. Smaller vessels become visible especially in the brain periphery (yellow arrows). Estimation of the spatial receive profile variations removed from both datasets for better visibility of smaller vessels.

6. Schmitter, et al: MRM.

7. Van de Moortele, et al: Proc. ISMRM. 2007, 1676.
doi:10.1186/1532-429X-14-S1-W72

Cite this article as: Schmitter et al:: Towards clinical application of $7 \mathrm{~T}$

TOF angiography. Journal of Cardiovascular Magnetic Resonance 201214

(Suppl 1):W72.

\section{Submit your next manuscript to BioMed Central} and take full advantage of:

- Convenient online submission

- Thorough peer review

- No space constraints or color figure charges

- Immediate publication on acceptance

- Inclusion in PubMed, CAS, Scopus and Google Scholar

- Research which is freely available for redistribution 